\title{
Two-dimensional photonic bandgap defect laser
}

\author{
O. Painter, R. K. Lee, A. Yariv, and A. Scherer \\ California Institute of Technology, Electrical Engineering, \\ MS 196-93, Pasadena, CA 91125, USA \\ $E$-mail: opainter@cco.caltech.edu \\ J. D. O'Brien, I. Kim, and P. D. Dapkus \\ University of Southem California, Department of Electrical Engineering, \\ Los Angeles, CA 90089
}

\begin{abstract}
With the maturation of crystal growth along with the nanofabrication of semiconductors, there has been strong interest in creating optical microcavities for spontaneous emission control. The Vertical Cavity Surface Emitting Laser(VCSEL) was the first device to shrink the optical mode to sizes on the order of the wavelength of light[1]. Subsequently, the microdisk laser was developed which uses total internal reflection to form high-Q whispering gallery modes[2]. In this work we form a new type of optical microcavity using two-dimensional photonic crystals embedded in a half wavelength thick waveguide. Modes localized to a single defect in the photonic crystal can be theoretically shown to have mode volumes as small $2(\lambda / 2 n)^{3}[3]$. The flexibility in design of the photonic crystal enables one to tailor the device for vertical emission or for coupling into an in-plane waveguide. The added versatility in being able to etch the laser cavity may also help develop low threshold laser sources in material systems in which high index contrast epitaxial mirrors do not exist.
\end{abstract}

Two dimensional(2D) photonic crystals have been fabricated and characterized in a variety of semiconductor materials, however they are not effective, by themselves, in confining optical modes in the third direction. In order to localize the light in all directions we use a combination of a microdisk type structure and a 2D photonic crystal. A thin dielectric slab is used for total-internal reflection of the light in the vertical direction, and a hexagonal array of air holes forms the 2D photonic crystal which provides in-plane localization. The microcavity structures are fabricated in the InGaAsP material system in order to reduce non-radiative surface recombination. Gain is provided by four strained InGaAsP quantum wells (QW) separated by quaternary barriers. The emission wavelength of the $\mathrm{QWs}$ are designed for $1.55 \mu \mathrm{m}$ at room temperature.

The optical cavity itself is created by removing a single hole in the photonic crystal, thereby forming an optical mode localized to the defect region[4]. The modes of the defect cavity have been analyzed previously [3] and consist of a pair of doubly degenerate $\mathrm{x}$ and $\mathrm{y}$ dipole modes. The electric field intensity of the y-dipole mode is shown in Figure 1.

The laser cavities were optically pumped with a $850 \mathrm{~nm}$ diode laser beam focused to approximately a $4 \mu \mathrm{m}$ spot covering the defect region. The photoluminescence was collected from above using a microscope objective and then run through a spectrometer. Pulsed lasing action was observed at a substrate temperature of 143K. A spectrum of the laser line just above threshold is shown in Figure 2 along with a plot of power at the lasing wavelength versus absorbed pump power. The relatively large threshold pump power can be attributed to the lack of thermal heat-sinking in the undercut membranes, which has so far limited lasing action to low temperature, pulsed operation. 
Two-Dimensional photonic bandgap..., 0 . Painter, et. al.

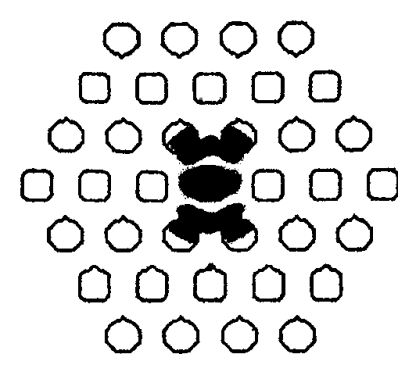

Figure 1: 2D slice through the middle of the slab showing the electric field amplitude of the $y$-dipole mode.

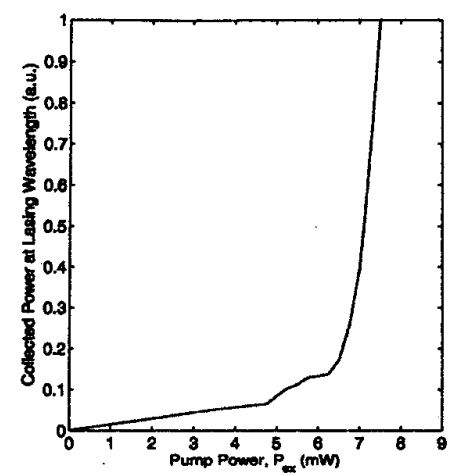

(a) L-L curve showing the power at the laser wavelength versus the absorbed pump power.

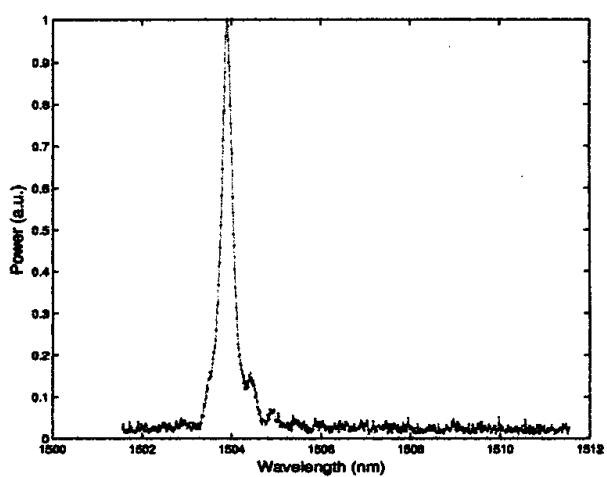

(b) Spectrum of the laser line just above threshold. The linewidth is approximately $0.2 \mathrm{~nm}$ (resolution limit of spectrometer).

Figure 2: Single mode defect laser at a substrate temperature of $143 \mathrm{~K}$

\section{References}

[1] J. L. Jewell, J. P. Harbison, A. Scherer, Y. H. Lee, and L. T. Florez, "Vertical-Cavity Surface-Emitting Lasers: Design, Growth, Fabrication, Characterization," IEEE Journal of Quantum Electronics 27, 1332-1346 (1996).

[2] A. F. J. Levi, S. L. McCall, S. J. Pearton, and R. A. Logan, "Room Temperature Operation of Submicrometre Radius Disk Laser," IEEE Electronics Letters 29, 1666-1667 (1993).

[3] O. Painter, J. Vuckovic, and A. Scherer, "Defect Modes of a Two-Dimensional Photonic Crystal in an Optically Thin Dielectric Slab," Journal of the Optical Society of America B 16, 275-285 (1999).

[4] P. R. Villeneuve, S. Fan, and J. D. Joannopoulos, "Microcavities in photonic crystals: Mode symmetry, tunability, and coupling efficiency," Physical Review B 54, 7837-7842 (1996). 